(HCV). September 21, 2001. http://www.fda.gov/bbs/ topics/ANSWERS/2001/ANS01103.html.

\section{Reuse of Single-Use Devices: FDA Delays Enforcement}

Hospitals that reprocess single-use devices (SUDs) have been given an extension on the date when the FDA will begin enforcing its new reprocessing requirements covering medical-device reporting, tracking, corrections and removals, quality system, and labeling. The extended deadline is August 14, 2002.

Hospitals still are required to register with the FDA and to list the SUDs they plan to reprocess. The FDA shortly will begin inspecting hospitals to assess their compliance with the requirements, but it intends to use those inspections as an opportunity to educate hospitals in the new requirements rather than to enforce actions. The education policy will be in effect until August 14, 2002 , "provided that the hospitals are taking steps to correct the violations noted in the inspection and that the violations do not pose a serious public health threat."

FROM: AHA news online; October 11, 2001. http:// www.AHA.org.

FDA, Center for Devices and Radiological Health. Changes in enforcement of FDA's requirements on reprocessing of single-use devices. http://www.fda.gov/cdrh/ reuse/reuse-letter-092501.html.

\section{Reuse of Single-Use Devices Not Widespread}

Two recent surveys of hospitals found that reuse of single-use devices is not widespread and that the majority of hospitals use third-party reprocessors. Nearly one half $(47.8 \%)$ of all respondents to an ECRI e-mail survey on single-use device (SUD) reuse reported that they will not allow any reuse in light of last year's FDA regulations. Most respondents did not change their decisions on reuse as the August 14, 2001, enforcement deadline approached; those who did change generally were less willing to reuse devices than they had been previously. Most facilities (86.4\%) that plan to continue reuse will do so exclusively through third-party reprocessors.

In a recent study completed by JCAHO for the FDA, hospitals reported reusing medical devices intended for single use at a relatively low rate. Of the 800 hospitals polled, $11 \%$ reuse SUDs and only $2 \%$ reuse class III devices posing the highest risk to patient safety. The most commonly used class III devices are electrodes used during angioplasty for coronary artery disease. Other reused class III devices include endotracheal tubes and angioplasty catheters. The report shows that $80 \%$ of hospitals reusing SUDs rely on commercial vendors registered and listed with the FDA to reprocess these devices. The study was conducted between September 2000 and March 2001 at hospitals of all sizes.
FROM: JCAHOnline. August 2001. http://www. jcaho.org/tip/jonline0801.html.

\section{Infection Rates in Low-Birth-Weight Neonates}

Zafar and coinvestigators from St Louis conducted a study to determine factors associated with an increasing rate of nosocomial infections (NIs) in infants with very low birth weights. They performed a retrospective review of clinical and NI databases for all infants with birth weights of $\leqslant 1,500 \mathrm{~g}$ admitted to an academic neonatal intensive care unit between January 1, 1991, and December 31, 1997 $(n=1,184)$. Two study periods were compared: 1991 to 1995 and 1996 to 1997. Among the 1,085 infants who survived beyond 48 hours, the proportion who developed NIs increased from $22 \%$ to $31 \%(P=.001)$, and the infection rate increased from 0.5 to 0.8 per 100 patient-days $(P<.001)$ during the period from 1996 to 1997. During that same period, the median duration of indwelling vascular access increased from 10 to 16 days $(P<.001)$, and the median duration of mechanical ventilation increased from 7 to 12 days $(P<.001)$. Although the device-specific rate of bloodstream or respiratory infections did not change, the increase in infections was directly attributable to the increasing proportion of infants who required these devices. In both study periods, the peak incidence of initial infection occurred between 10 and 20 days of age. For the entire sample, proportional hazard models identified birth weight, duration of vascular access, and postnatal corticosteroid exposure as significant contributors to the risk of infection.

The authors concluded that the increasing number of technology-dependent infants was the primary determinant in the increase of NIs. Because these infections occur in a small proportion of infants, understanding the host factors that contribute to this vulnerability is necessary to control NIs in neonatal intensive care units.

FROM: Zafar N, Wallace CM, Kieffer P, Schroeder P, Schootman M, Hamvas A. Improving survival of vulnerable infants increases neonatal intensive care unit nosocomial infection rate. Arch Pediatr Adolesc Med 2001;155:1098-1104.

\section{Efficacy of Surveillance for Infection Control in a Surgical Service}

Delgado-Rodriguez and colleagues, from the University of Jaen in Spain, conducted a study to assess the efficacy of surveillance of nosocomial infection (NI) in infection control at a service of general surgery. A surveillance study that included 1,483 patients with a prospective identification of NI was carried out. Its results were discussed with the staff, and a program on NI control was implemented. One year after the pre-intervention study, a similar study that included 1,506 patients was done. The main outcome measure was NI. Incidence rates, incidence rate ratios, crude and multiple-risk factor adjusted for by 